\title{
Citizen science and food
}

\author{
Area of research interest: Behaviour and perception
}

Citizen science and food is part of a new programme of work to explore how we can involve the communities we serve when building the evidence-base on which policy decisions are made.

Citizen science is an approach that can provide high volumes of data with a wide geographic spread. It is relatively quick to deploy and allows access to evidence we would ordinarily have difficulty collating.

This methodology has been endorsed by the European Commission for Research, Science and Innovation. There is no one size fits all definition, but citizen science projects involves engaging with communities and asking them to be part of the project, either through engaging them in data collection or through other ways of co-creation.

For participants, citizen science offers learning opportunities, the satisfaction of contributing to scientific evidence and the potential to influence policy. It can also give us data which is high in volume, has wide geographical spread, is relatively quick to deploy and that we couldn't access any other way. Projects using these methods often involve engaging with communities and asking them to be part of the project. This can be either through working with them in data collection, or through co-creation.

This report demonstrates that the research community are already undertaking numerous pieces of research that align with FSA's evidence needs. This includes examples from the UK and other global communities. Participants in such research have collected data on topics ranging from food preparation in the home to levels of chemical contaminant in foods.

The findings of this report outline that citizen science could allow the FSA to target and facilitate more systematic engagement with UK and global research communities, to help address key research priorities of the FSA.

Research report

PDF

View Citizen Science and Food - A Review as PDF(Open in a new window) (845.74 KB) 